\title{
GAIA Maternal Immunization Level of Diagnostic Certainty
}

National Cancer Institute

\section{Source}

National Cancer Institute. GAIA Maternal Immunization Level of Diagnostic Certainty. NCI

Thesaurus. Code C126867.

A classification of maternal and fetal outcomes relating to maternal immunization, developed by the Global Alignment of Immunization safety Assessment in pregnancy, based on the extent to which the diagnosis has been confirmed. 